\title{
Session VI
}

\section{Galaxy Formation}




\title{
Current status of galaxy formation modelling
}

\author{
Patricia B. Tissera ${ }^{1}$ \\ ${ }^{1}$ Institute for Astronomy and Space Physics, CC 67, Suc.28, Buenos Aires, Argentina \\ email: patricia@iafe.uba.ar
}

\begin{abstract}
I summarize the main characteristics of cosmological galaxy formation models and discuss some of the main results obtained in the last years.
\end{abstract}

Keywords. galaxy:formation, galaxy:evolution

\section{Introduction}

The ever growing observational data on galaxies and their stellar populations either in the nearby universe or at high redshift open an unprecedent opportunity to study galaxy formation. Cosmological simulations are a powerful tool since they can describe the joint evolution of dark matter and baryons self-consistently and provide a way of interpreting observations gathered at different redshifts. Numerical codes can be Eularian or Lagrangian. They are both efficient depending on the problem to be tackled. And in the last decades, there have been large improvements in the optimazation and parallelization of codes written under either approach. I apologize this review will be biased towards simulation results based on the Smooth Particle Hydrodynamics (SPH) which is a Lagrangian technique proven to be specially convenient for the study of galaxy formation in a cosmological context.

The cosmological framework is currently established with high precision by observations (e.g. Sánchez et al. 2006). In this scenario, the structure grows hierarchically from primordial fluctuations via gravitational instability. At large scales, the mass is distributed in a filamentary fashion with groups and clusters forming at the nodes of this so-called cosmic web. This evolution can be followed by integrating the gravitational field which is quite efficiently carried out by cosmological codes (e.g Frenk et al. 1998). In fact, $\Lambda$-CDM cosmology has passed successfully many tests on the large-scale distribution of the matter. At galaxy-scales, dark matter haloes have also been exhaustively studied. Navarro et al. (1996) first claimed them to have an universal density profile. Higher numerical resolution simulations have shown that the way in which galaxies are assembled plays a key role in shaping the final dark matter distribution in haloes, imprinting particularities which make them departure from an universal density profile (Navarro et al. 2009).

The main challenges arise when baryons are included in the models. The description of the structure formation in a cosmological scenario together with the relevant physics affecting the evolution of baryons is extremely difficult since the spatial and temporalscales involved are very different. In the last years, large efforts have been made in order to develop models for the main physical processes governing the formation of galaxies (e.g. Katz \& Gunn 1991; Cen \& Ostriker 1992; Navarro \& White 1993; Metzler et al. 1994; Gerritsen \& Icke 1997; Yepes et al. 1997; Sommer-Larsen et al. 1999; Thacker \& Couchman 2001; Kay et al. 2002; Semelin \& Combes 2002; Abadi et al. 2003; Springel \& Hernquist 2003; Okamoto et al. 2003; Scannapieco et al. 2005; Keres et al. 2005; 
Oppenheimer \& Davé 2006; Stinson et al. 2006; Governato et al. 2007; Dalla Vecchia \& Schaye 2008; Dubois \& Teyssier 2008; Filantor \& Davé 2008; Scannapieco et al. 2008).

State-of-arts models include treatments for radiative cooling, star formation and Supernova (SN) feedback, among others. Numerical resolution limits how far physical processes can be described reliably in simulations. Because of this, sub-grid models for the small-scale physical processes have been started to be developed. In general words, sub-grid modelling follows analytically the physics on the scales that cannot be resolved and then translate their effects to scales that can actually be numerically well-described. The efforts to include more physics in the modelling have been rewarded and general improvements have been achieved. Current models follow the formation of galaxies describing the dynamical and chemical evolution of the stellar populations and the interstellar medium. Of course, much work is still needed in many open aspects which will be surely matter of study in the next decade.

\section{Modelling the stellar populations in cosmological simulations}

The understanding of the observed Universe and the self-consistent concatenation of observations obtained at different redshift demands the development of sophisticated models which can describe not only the gravitational growth of the structure but the complex evolution of baryons. As we have already mentioned, the relevant scales for the physics of baryons are either marginally solved or directly unresolved. Hence, schematic or sub-grid modelling are used instead to describe several physical processes.

As the dark matter haloes are formed, baryons in the form of gas are allowed to cool down. The main cooling process is the bremstralung radiative cooling. Most authors adopt the radiative rates of Sutherland \& Dopita (1995). As it is known, the rate of radiated energy is a function of temperature and metallicity. In fact, the rates can vary several order of magnitudes for different gas metallicities at a given temperature. Hence, it is very important for the cosmological models to follow the chemical enrichment of baryons. Some codes even compute radiative cooling element-by-element (e.g. Wiersma et al. 2009).

In dense and cold gas, molecular clouds should form provided the feeding material for the star formation process. Due to the fact that this process occurs at scales smaller than those that current numerical resolution can resolve, a series of constrains are adopted to select gas clouds with high probability to be star-forming regions. In general, cold and dense gas clouds are considered to be suitable sites for star formation if certain thresholds on density and temperature are fullfiled. Most of the codes assumes a motivated SchmidtKennicutt law to relate the star formation rate with the gas density (see Schaye \& Dalla Vecchia 2008 for an extensive discussion on this particular point).

Since, on one hand, the cooling time of a gas cloud depends inversely on its density and, on the other hand, the rate of transformation of gas into stars depends directly on cold gas density, star formation can turn out to be very efficient if no effective heating processes are included (e.g. Weil et al. 1998). A catastrophic cycle could lead from a very efficient gas cooling to a very efficient star formation activity which might exhaust the gas reservoirs of the galactic systems at early times, overproducing spheroidal systems dominated by old stellar populations.

The interstellar medium (ISM) has a very complex structure which cannot be well described yet by cosmological models. In particular, the SPH technique tends to oversmooth the density field so that if the hot and diffuse gas component is located nearby a cold gas cloud, the former will have its density overestimated by the contribution of these cold and dense neighbouring clouds to the calculations. The increase of the gas 
density leads to shorter cooling times, with the consequence of increasing the fraction of baryons in the cold and dense phase which, at the end, will very easily satisfy the star formation constrains. A more realistic representation of the multi-phase character of the ISM which allows the coexistence of gas clouds with different thermodynamical properties would contribute to alleviate this problem (e.g. Yepes et al. 1997; Marri \& White 2003; Scannapieco et al. 2006).

SN events are considered a very effective mechanism capable of regulating the rate of gas transformation into stars. During the last decades, enormous efforts have been devoted to the search of a self-consistent model. Katz (1992) showed that pumping the ejected energy directly into the thermal component has no significant effects on the gas dynamics because this energy is quickly radiated away. This can be understood considering that SNII progenitors have short lifetimes which in practice, results in the release of the energy in the same regions where the progenitors stars have formed. These regions are cold and dense and, consequently, have very short cooling times.

In an attempt to solve this problem, the so-called kinetic or momentum-driven feedback was proposed (Navarro \& White 1993). In this model, the gas particles surrounding the sites of SN explosions are directly given kinetic energy, imprinting a spatial displacement and a net change in the velocity of the gas. The advantage of this scheme is that it produces the desired effect: there is a net change in dynamics of the gas component and, as a consequence, the star formation activity can be regulated and large-scale outflows, driven. The disadvantages are the non-physical basis of the model and the introduction of scale-dependent parameters, which force the model to have a characteristic mass or velocity. Nevertheless, an important effort has been invested to determine these scaledependent parameters on more physical basis as discussed by Oppenheimer \& Davé (2006), for example.

A different approach is proposed by Stinson et al. (2006) who disabled the radiative cooling of gas particles in the surrounding region where SNII events are produced, for a certain time interval. The size of this region and this time interval are estimated by applying the blast-wave analytical treatment of McKee \& Ostriker (1977) which allows their determination on more physical basis.

The last approach we are going to discussed is that proposed by Scannapieco et al. (2006) based on the previous work of Marri \& White (2003). In this scheme the SN feedback works together with a multiphase model for the interstellar medium which allows the coexistence of gas clouds of different temperature and density (i.e. entropy) without introducing scale-dependent parameters. Energy and chemical elements produced by SNII and SNIa are distributed in the ISM by defining cold and hot environments associated to the simulated HII regions. In Fig. 1 we can see the surface gas density of a MilkyWay type galaxy simulated without and with SN feedback (the maps have $20 \mathrm{kpc} h^{-1}$ length side). In the first case, the gas component forms a very concentrated spheroidal system (the stars also follow this distribution) while in the second, when SNe are taken into account, the gas is able to form a disc. The ISM of this disc shows a more complex structure in the density (and temperature) distribution. This model is successful at triggering galactic outflows which reproduce observed trends as well as regulating the star formation activity during quiescent periods of evolution. It is suitable to study galaxy formation within a cosmological framework since there is no scale or mass-dependent parameters.

Following the chemical enrichment of baryons together with the dynamical growth of the structure is a powerful tool to constrain galaxy formation models. Analytical chemical models have provided proofs of the key role played by chemical elements to the understanding of the galaxy formation (e.g. Chiappini et al. 1997). In cosmological 
simulations, chemical evolution has been included in the last years (e.g., Raiteri et al. 1996; Mosconi et al. 2001; Lia et al. 2002; Kawata \& Gibson 2003; Okamoto et al. 2003; Scannapieco et al. 2005). Most of current models considers both SNIa and SNII chemical production with different levels of sophistication. SNII are assumed to originate from massive stars with lifetimes of the order of $10^{6}-10^{7}$ yrs, while SNIa are generally considered to originate from binary systems with lifetime of the order of a Gyr. This difference in the life-times produces a differential enrichment of baryons since SNIa is the main producer of Fe while $\alpha$-elements are mainly produced by SNII. Hence, the relative abundance of $\alpha$-elements to Fe is taken as a strong constrain for the baryonic history of the system. Note that baryonic history implies the star formation, the cold gas accretion and outflows histories all together and it is grafted into the assembled history of a galaxy. As we can image it is very complicated to disentangle the relative importance of each of them from the resulting baryonic history and chemical patterns. And, indeed, this is where numerical simulation can make an important contribution. The outcomes of these models provide the dynamical and chemical properties of the stellar population and the ISM in the simulated galaxies and their corresponding evolution in a cosmological framework. To confront them more robustly with observations, luminosities can be estimated by combining the stellar population synthesis models with the information of the age and metallicity of different simulated stellar population (e.g. Tissera, Lambas \& Abadi 1997).

\section{Results and open problems}

Galaxy formation models are used to study different aspect of this problematic. Particularly, the formation of spiral galaxies has remained as one of the most challenging problems for the hierarchical clustering scenario and galaxy formation models, although in the last years, there has been important progress. There are two main problems which conspired against the standard disc formation picture (Fall \& Efstathiou 1980). First, gas

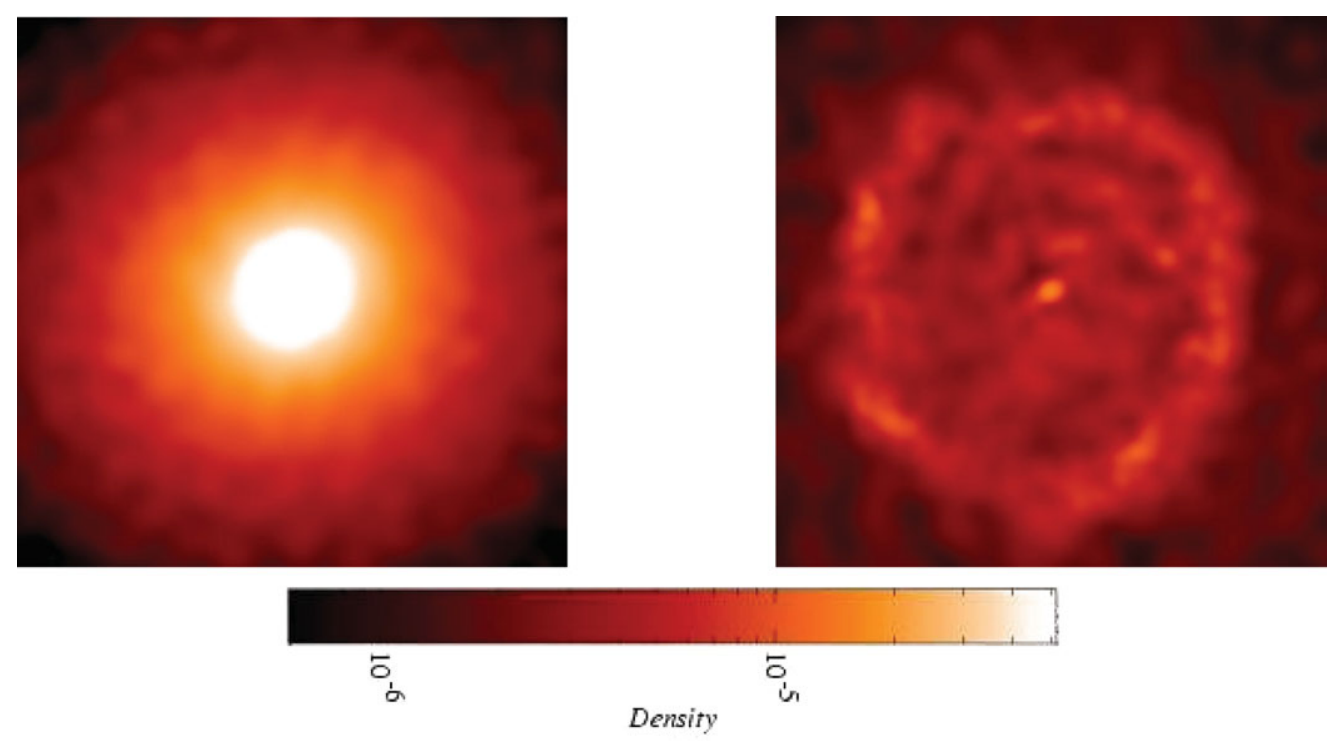

Figure 1. Surface density maps of the gas components for a galaxy simulated without (left panel) and with (right panel) SN feedback. The self-consistent treatment for SN feedback allows the description of a multiphase gas where clouds of different temperature and density can more easily coexist. Density in units of $10^{10} \mathrm{M}_{\odot} \mathrm{kpc} \mathrm{h}^{-1}$. 
cooling is very efficient and leads to an early consumption of the gas reservoirs required for the formation of thin discs. And secondly, discs are unstable structures which can be destabilized quite easily during accretion of satellites and interactions with nearby systems. In a hierarchical scenario, accretion of satellites is an ubiquitous process which is more frequent at high redshift. When a disc is disrupted the material can be easily fueled into the central regions, contributing to the formation of a bulge component (Scannapieco et al. 2009). This gas is shocked and compressed becoming suitable for star formation. Unless there are efficient mechanisms to regulate the transformation of gas into stars, this process will consume an important fraction of the galaxy gas reservoirs, preventing the rebuilding of a new disc, afterwards.

The improvement in the SN feedback modelling has allowed the formation of disc+ bulge systems which resemble Sa/Sb galaxies (Governato et al. 2007; Scannapieco et al. 2008). However, pure disc structures have remained elusive. As shown by Scannapieco et al. (2008) SN feedback is a key process which contributes to the formation of disc structures because it regulates the star formation activity, inhibiting the early transformation of gas into stars in the progenitors systems and the infalling satellites. An important fraction of gas is expelled by the triggered galactic winds and later on, adquired by the main galaxy forming a disc. This gas is a reservoir of angular momentum (see also Pedrosa et al. 2010). In Fig. 2 we can see the how the strength of the estimated galactic wind correlates with disc-to-spheroid mass ratio, indicating the important role of galactic winds in the formation of disc-like structures. When disc systems are able to form, they follow the expected relation between stellar mass and circular velocity in agreement with observed Tully-Fisher relation (e.g. Governato et al. 2007). Recently, De Rossi et al. (2009a) estimated the stellar mass and baryonic mass Tully-Fisher relation at $z=0$ and their evolution with redshift. These authors showed the key role played by the SN feedback in the regulation of the SF activity and the gas content of the systems via the galactic winds which worked together to yield a relation in agreement with observations.

As baryons are accreted onto the dark matter haloes forming galaxies of different morphologies, the dark matter gets compress. To account for this process, the adiabatic contraction hypothesis was used for many years. Models such as that of Blumenthal et al. (1986) have proven useful to estimate the reaction of the dark matter distribution to the building up of galaxies. However, it is now clear that this hypothesis is an oversimplification of the process which leads to a larger effect than those reported by hydrodynamical simulations (e.g. Tissera \& Domínguez-Tenreiro 1998). It is clear that the presence of baryons increases the dark matter concentration, changing the shapes and velocity ellipsoids of haloes in a non-trivial way. Dark matter haloes are close to isothermal in the region where baryons dominate (Romano-Díaz et al. 2008) with a general trend to become triaxial (e.g. Tissera \& Domínguez-Tenreiro 1998; Kazantzidis et al. 2004). Recent results suggest that not only the merger history (Tissera et al. 2010 in preparation) but also the baryonic history are relevant in shaping the DM haloes as shown by Pedrosa et al. $(2009,2010)$.

It has been shown that the gas cooling and accretion onto the halo potential well could be more complex than previously thought. Keres et al. (2005) claimed the existence of two regimes for gas accretion (see also Birnboim \& Dekel 2003) to form a galaxy: the infall of cold gas along filaments which was found to be efficient up to a certain critical mass and the convential shocked virial-heated gas which is then able to cool down and condense forming a galaxy. This hot mode was reported to be more efficient for high mass haloes. In fact, recently Governato et al. (2009) claimed that the cold mode was the principal contributor to the star formation activity in a Milky-Way type galaxy. In a very interesting work, Ocvirk et al. (2008) studied the role played by metallicity as a 
key ingredient to describe the gas evolution and as a consequence, in the regulation of the star formation process.

\subsection{Chemical properties: the master key to constrain galaxy formation models?}

The chemical properties of stars and gas are also now available to confront observations and models. For example, the mass-metallicity relation is globally reproduced (e.g. Governato et al. 2007; Kobayashi et al. 2007; De Rossi \& Tissera 2009). However, the evolution of this relation is still a controversial issue for models and observations. Particularly, there are many uncertainties in the determination of the observed stellar masses and metallicities at high redshift which make difficult to reach a robust conclusion. Scannapieco et al. (2008) showed that if a disc is able to form, then the global metallicity trends detected in the bulge and disc of the Milky-Way are present. The stars on the simulated discs have a metallicity profile consistent with observed values and those in the halo and bulge components tend to exhibit more $\alpha$-enhanced than those on the disc component. As an example, in Fig. 3 we can see the $[\mathrm{Fe} / \mathrm{H}]$ distribution for the components: bulge, disc and halo of two galaxies in the set of 10 different haloes of $\approx 10^{12} \mathrm{M}_{\odot}$ ran by Scannapieco et al. (2009) as part of the Aquarius project. We can see that stars in the halo are Fe poor as expected while the stars in the bulge have larger chemical enrichment (Tissera et al. 2010, in preparation). But, it is also interesting to note how these two galactic systems, although having similar final masses, have different metallicity distributions. A challenging problem is to reliably quantify the abundance patterns which could be related to the merger and baryonic histories of formation of these galaxies.

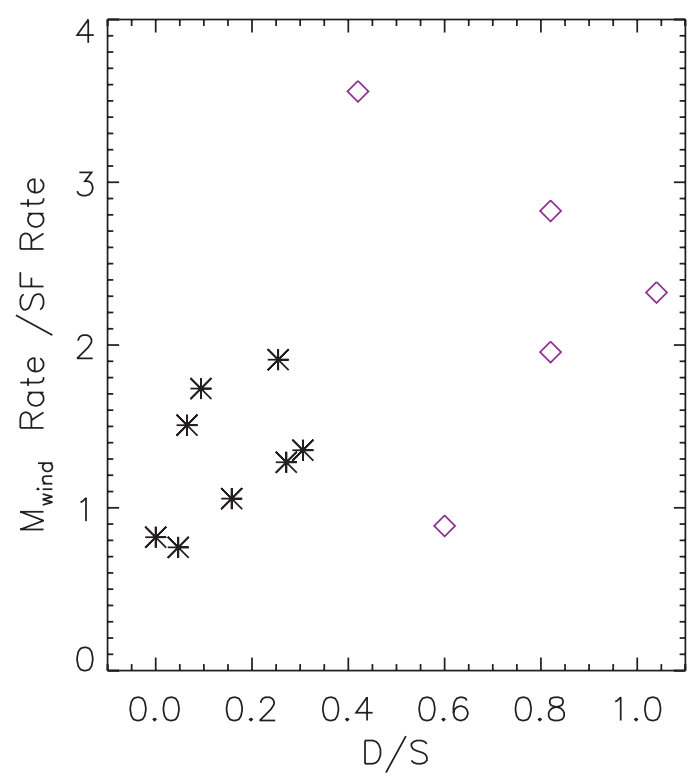

Figure 2. Galactic wind strength as a function of disc-to-spheroid mass ratio for simulated galaxies in Scannapieco et al. (2008)(diamonds) and for the simulated galaxies (asterisks) as part of the Aquarius Project (Scannapieco et al. 2009). The two outlying point at $D / S \approx 0.5-0.6$ correspond to very extreme parameters for the SF and SN models. 

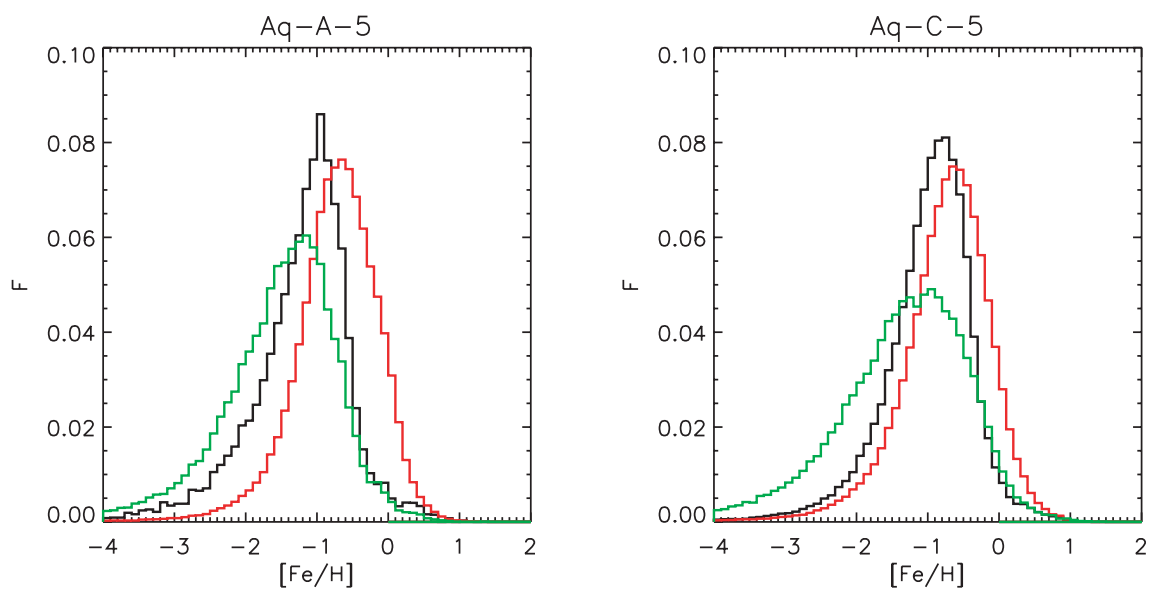

Figure 3. Distribution of $[\mathrm{Fe} / \mathrm{H}]$ for the stellar populations located in the central spheroid (red lines), the disc (black lines) and halo (green lines) of the two different $\approx 10^{12} \mathrm{M}_{\odot}$ haloes in the Aquarius project (Tissera et al. in preparation).

\section{An (incomplete) list of interesting and/or open points}

There are many challenging issues related to the modellization of stellar populations in cosmological simulations to work on in the next decades. To end up, I would like just to list some of them:

- Gas cooling and collapse is described by the models but we are just starting to understand how the gas flows along the filamentary structure.

- Star formation is still very simply modelled.

- SN feedback is better described but there is still much to understand.

- We have disc systems! But thin, bulge-less discs are still missing.

- We can describe the chemical enrichment of baryons and this will be an important tool to constrain galaxy formation. Chemical mixing is still probably not properly represented.

- The physics to describe the formation of first stars remains a very interesting area where several authors have made important improvements (e.g. Bromm et al. 2009) and we will have important news in the next decade.

- There are many open questions regarding AGN feedback related to the physics and to its modellization in cosmological models (e.g. Puchwein et al. 2010) .

- Improvement needed for the comparison pipeline with observations and for the statistics in simulations.

- Improvement needed for sub-grid modelling and numerical resolution.

\section{Acknowledgements}

PBT thanks M.E. De Rossi, S. Pedrosa and M.J. Perez for useful comments of this manuscript. PBT thanks the L'oreal prize, PICT N 245 (2006) Max Planck and the organizers of the meeting which partially supported this review.

\section{References}

Abadi M. G., Navarro J. F., Steinmetz M., \& Eke V. R. 2003, ApJ, 591, 499

Birnboim, Y. \& Dekel, A. 2003, MNRAS, 345, 349

Blumenthal,G. R., Faber, S. M., Flores, R., \& Primack, J. R. 1986, ApJ, 301, 27

Bromm, V., Yoshida, N., Hernquist, L., \& Mckee, C, F. 2009, Nature, 459, 49 
Cen, R. \& Ostriker, J. P. 1992, ApJ, 399, L113

Cen, R. \& Ostriker, J. P. 1999, ApJ, 519, L109

Chiappini, C., Matteucci, F., \& Gratton, R 1997, ApJ, 477, 765

Dalla, Vecchia, C., \& Schaye, J. 2008, MNRAS, in press (astro-ph/0801.2770)

De Rossi, M. E., Tissera, P. B., \& Pedrosa, S. 2009a, MNRAS, submitted

De Rossi, M. E., Tissera, P. B., \& Pedrosa, S. 2009b, Proceeding IAU S262: Stellar Populations: Planning for the next decade, Rio de Janeiro, 2009

De Rossi, M. E., Tissera, P. B., \& Pedrosa, S. 2009c, Proceeding of 52 Annual Meeting of the Argentinian Astronomical Society, La Plata, Buenos Aires, 2009

De Rossi, M. E. \& Tissera, Proceeding IAU S262: Stellar Populations: Planning for the next decade, Rio de Janeiro, 2009

Dubois, Y. \& Teyssier, R. 2008, A\&3A, 477, 79

Eke, V. R., Efstathiou, G., \& Wright, L. 2000, MNRAS, 315, 18L

Fall, S. M. \& Efstathiou, G. 1980, MNRAS, 193, 189

Finlator, K. \& Davé, R. 2008, MNRAS, 385, 2181

Frenk, C., S., White, S.D. M., Davis, M., \& Efstathiou, G. 1998, ApJ, 227, 507

Gerritsen, J. P. E. \& Icke, V. 1997, A\& A, 325, 972

Governato, F., Willman, B., Mayer, L., Brooks, A., Stinson, G., Valenzuela, O., Wadsley, J., \& Quinn, T. 2007, MNRAS, 374, 1479

Governato F., et al. 2004, ApJ, 607, 688

Governato, F. Brook, C. B., Brooks, A. M., Mayer, L., Willman, B., Jonsson, P., Stilp, A. M. et al. 2009, MNRAS, submitted

Katz, N. 1992, ApJ, 391, 502

Katz, N. \& Gunn, J. E. 1991, ApJ, 377, 365

Kay, S. T., Pearce, F. R., Frenk, C. S., \& Jenkins A. 2002, MNRAS, 330, 113

Kazantzidis, S., Mayer, L., Mastropietro, C., Diemand, J., Stadel, J., \& Moore, B. 2004, ApJ, 608,663

Keres, D., Katz, N., Weinberg, D. H., \& Davé, R. 2005, MNRAS, 363, 2

Kobayashi, C., Springel, V., \& White, S. D. M. 2007, MNRAS, 376, 1465

Lia, C., Portinari, L., \& Carraro, G. 2002, MNRAS, 330, 821

Marri, S. \& White, S. D. M. 2003, MNRAS, 345, 561

Matteucci, F. 2008, to appear on the Proceedings of the 37th Saas-Fee Advanced Course of the Swiss Society for Astrophysics and Astronomy, "The Origin of the Galaxy and the Local Group", eds. E. Grebel and B. Moore.

McKee, C. F. \& Ostriker, J. P. 1977, ApJ, 218, 148

Metzler, C. A. \& Evrard, A. E. 1994, ApJ, 437, 564

Mosconi, M. B., Tissera, P. B., Lambas, D. G., \& Cora, S. A. 2001, MNRAS, 325, 34

Navarro, J. F. \& White, S. D. M. 1993, MNRAS, 265, 271

Navarro, J. F. \& White, S. D. M. 1994, MNRAS, 267, 401

Navarro, J. F., Frenk, C., \& White S. D. M. 1997, ApJ, 490, 493

Navarro, J. F., Ludlow, A., Springel, V.., Wang, J., Vogelsberger, White S. D. M., M., Jenkins, A., Frenk, C. S., \& Helmi, A. 2008, MNRAS, submitted

Ocvirk, P., Pichon, C., \& Teyssier, R. 2008, MNRAS, 390, 1326

Okamoto, T., Jenkins, A., Eke, V. R., Quilis, V., \& Frenk, C. S. 2003, MNRAS, 345, 429

Oppenheimer, B. D. \& Davé R. 2006, MNRAS, 373, 1265

Pedrosa, S., Tissera, P. B., \& Scannapieco, C. 2009, MNRAS, 395, L57

Pedrosa, S., Tissera, P. B., \& Scannapieco, C. 2010, MNRAS, submitted

Puchwein, E., Sijacki, D., \& Springel, V. 2010, To appear in proceedings of the conference The Monster's Fiery Breath: Feedback in Galaxies, Groups, and Clusters, June 2009, Madison, Wisconsin.

Raiteri, C. M., Villata, M., \& Navarro, J. F. 1996, A\&A, 315, 105

Romano-Díaz, E., Shlosman, I., Heller, C., \& Hoffman, Y. 2008, ApJ, submitted

Schaye, J. \& Dalla Vecchia, C. 2008, MNRAS, 383, 1210

Scannapieco, C., Tissera, P. B., White, S. D. M., \& Springel, V. 2005, MNRAS, 364, 552

Scannapieco, C., Tissera, P. B., White, S. D. M., \& Springel, V. 2006, MNRAS,371, 1125 
Scannapieco, C., Tissera, P. B., White, S. D. M., \& Springel, V. 2008, MNRAS, 389, 1137

Scannapieco, C. , White, S. D. M., Springel, V., \& Tissera, P. B. 2009, MNRAS, in press

Semelin, B. \& Combes, F. 2002, A\&A, 388, 826

Sommer-Larsen, J., Gelato, S., \& Vedel, H. 1999, ApJ, 519, 501

Springel, V. \& Hernquist, L. 2003, MNRAS, 339, 289

Springel, V. 2005, MNRAS, 364, 1105

Springel,,, V., 2010, MNRAS,

Stinson, G., Seth, A., Katz, N., Wadsley, J., Governato, F., \& Quinn, T. 2006, MNRAS, 373, 1074

Sutherland, R. S. \& Dopita, M. A. 1993, ApJS, 88, 253

Thacker, R. J., Couchman, H. M. P. 2001, ApJ, 555, L17

Tissera, P. B., Lambas, D. G., \& Abadi, M. 1997, MNRAS, 286, 384

Tissera, P. B . \& Domínguez-Tenreiro, R. 1998, MNRAS, 297, 177

Weil, M. L., Eke, V. R., \& Efasthaiou, G. 1998, MNRAS, 300, 773

Wiersma, R. P. C., Schaye, J., Theuns, T., Dalla Vecchia, C., \& Tornatore, L. 2009, MNRAS, in press

Yepes, G., Kates, R., Khokhlov, A., \& Klypin, A. 1997, MNRAS, 284, 235

Zoccali, M., et al. 2003, A\& A, 399, 931 